\title{
A New Representation System in Tonal Harmony for Analysis and Creation
}

\author{
Anna Shvets \\ Maria Curie-Sklodowska University \\ Plac Marii Curie-Sklodowskiej 5, Lublin, Poland \\ annashvets11@gmail.com
}

\section{INTRODUCTION}

Contemporary music theory development reflected transition inherent to the information society from substantial, object-oriented analysis, to the transformational network. This development is presented in succession of the theories based on neo-Riemannian approach and Tonnetz as a space for possible connections establishment.

\section{A NEW REPRESENTATION SYSTEM}

A new system of music harmony representation proposes an alternative space for establishment of the connection patterns. The system is based on the functional harmonic logic and uses graph theory for construction of the frame units: a basic proto-frame structure unfolds to the system of exoframes presented as graphs. Grouping of the frame instances (representing harmonic progressions) based on their resemblance creates a higher structural level of the harmony logic. The received graph patterns may be further expressed graphically, with a unified system of shapes (schemas), or logically, in alphabetic representation. The schema representation allows us to represent the process of harmonic development of the whole composition in one range of graphical shapes placed on the time line. The structural harmony method with the two-level representation opens huge possibilities for comparison of a large amount of the frame instances represented in a very concise manner.

\section{SCHEMOGRAPHE APPLICATION}

The possibilities of the interactive harmonic visualisation using elaborated system of the structural harmony method are presented using developed Schemographe android application for neo-tonal music representation (Shvets \& Desainte-Catherine 2015). The application contains the graphical representation of harmonic analysis made out of three vocal pieces by postmodern composer Valentin Silvestrov. The first view of the application gives an access to each of the analysed pieces through buttons (Figure 1); after the specified piece have been chosen, its harmonic development in schemas representation will appear on the screen (Figure 2). Each schema item is interactive and leads to the graph representation of the harmonic pattern shown in a process of building with appropriate schema features formation and is related to the music events (Figure 3 ). The general representation of all the schemas for all pieces is available in generalised view (Figure 4), accessible through the appropriate button on the main view.

\section{MODULE OF THE HARMONIC SEQUENCES CONSTRUCTOR APPLICATION}

The display of the schema structures with an access to the graph patterns may be reversed in order if harmonic sequences are created by user. A module of such software for android based tablets shows the possibilities of the structural harmony method applied to the music creation proposing an easy-to-use graphical interface. The module consists of two views: the view representing a part of a graph system in which user can choose which graph he/she wants to explore (Figure 5) and the view allowing harmonic sequence construction itself (Figure 6).

\section{REFERENCES}

Shvets, A. \& Desainte-Catherine, M. (2015) Schemographe: Application for a New Representation Technique and Methodology of Analysis in Tonal Harmony. Evolutionary and Biologically Inspired Music, Sound, Art and Design. Springer, Lecture Notes in Computer Science, Volume 9027, pp. 212-223. 


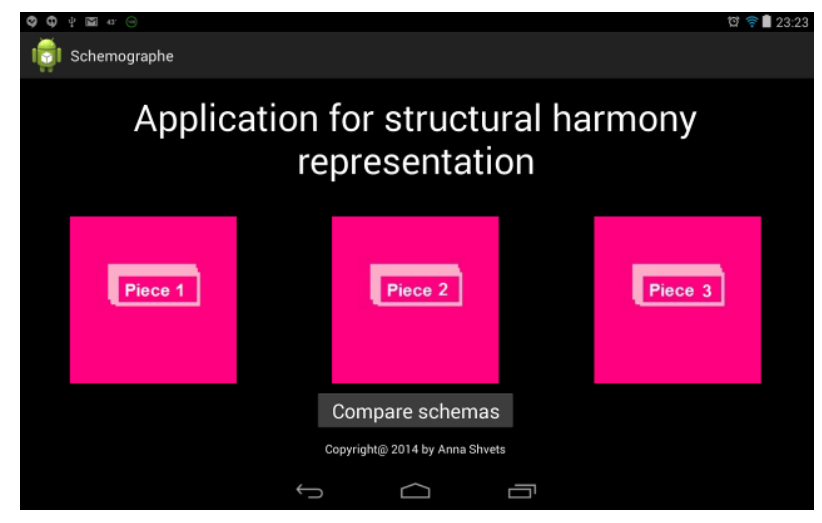

Figure 1: Schemographe: main view

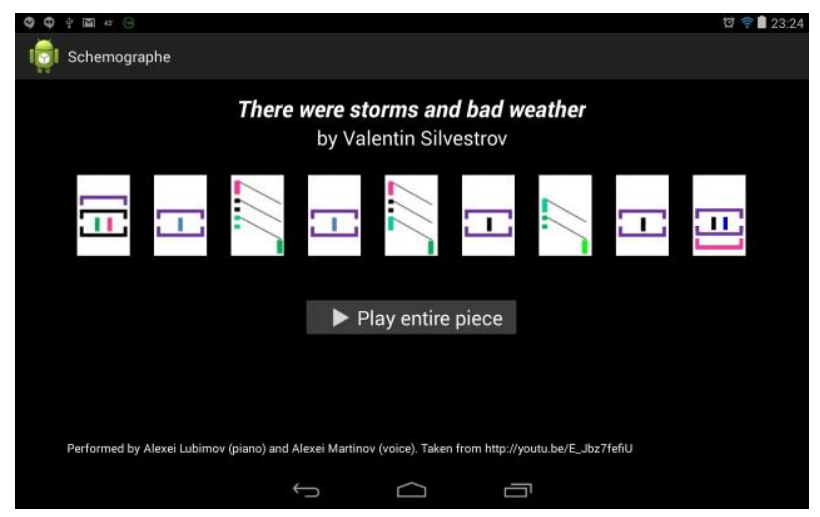

Figure 2: Schemographe: schemas of the selected piece view

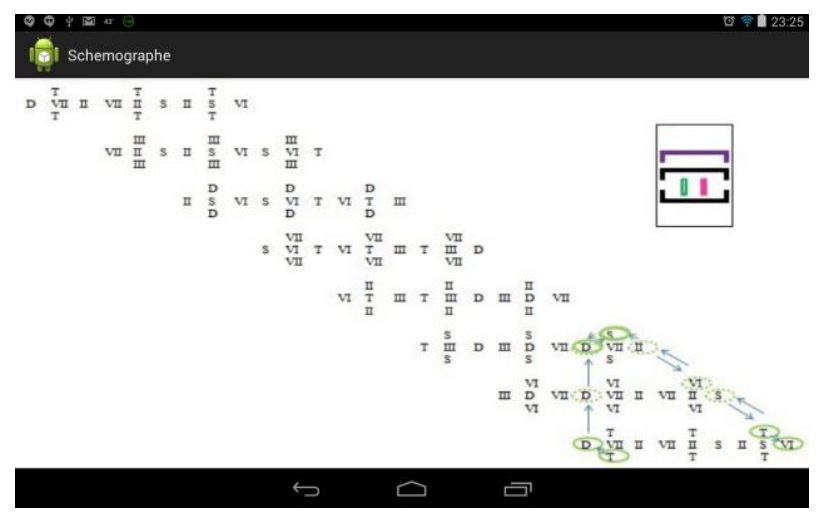

Figure 3: Schemographe: graph pattern formation of the selected schema

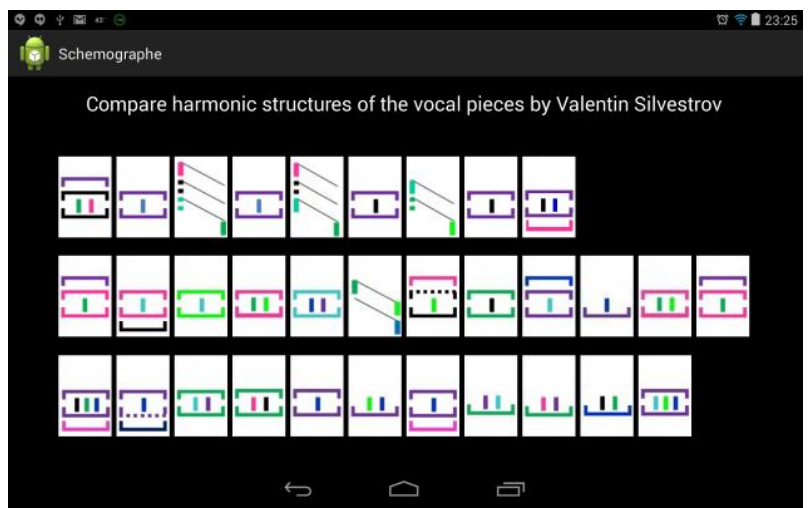

Figure 4: Schemographe: generalised view of all the schemas from all the pieces

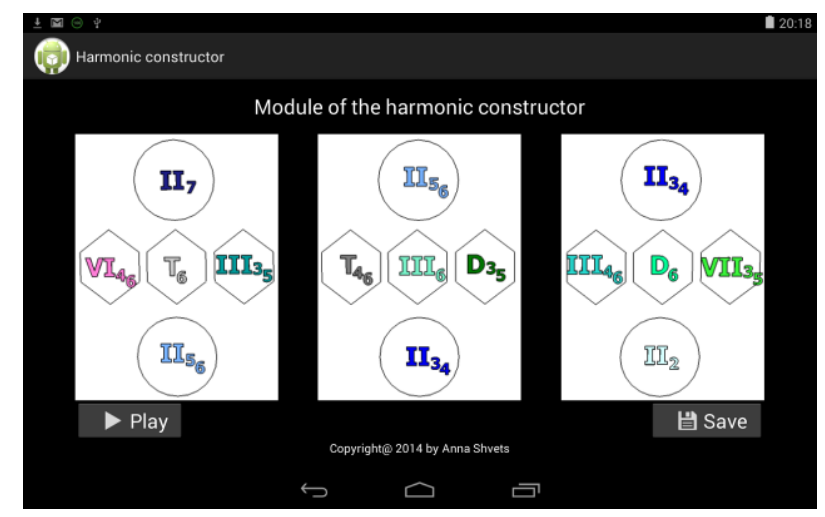

Figure 5: Module of the harmonic constructor: main view

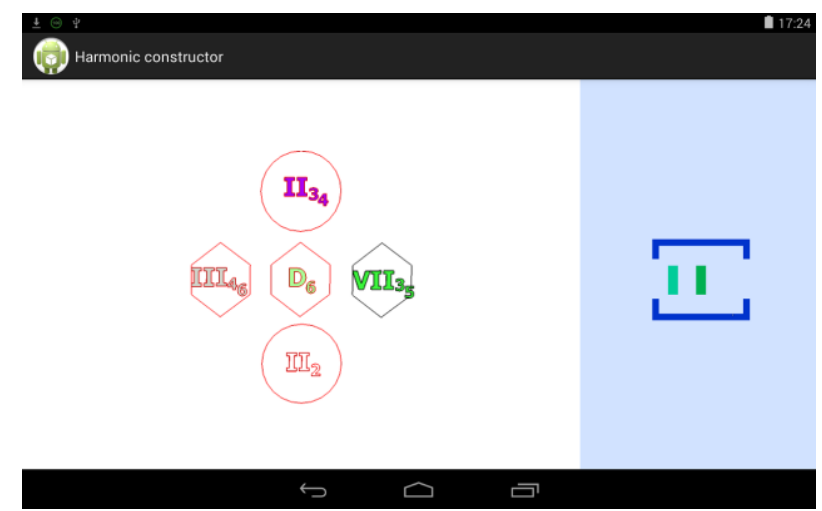

Figure 6: Module of the harmonic constructor generation: individual graph formation view 